\title{
Process tracing. InduCCión, Deducción E INFERENCia CAUSAL ${ }^{*}$
}

Process Tracing: Induction, Deduction, and Causal Inference

\author{
TOMAS BRIL-MASCARENHAS \\ Escuela de Política y Gobierno, Universidad Nacional de San Martín (UNSAM), Argentina
}

\author{
ANTOINE MAILLET \\ Instituto de Asuntos Públicos, Universidad de Chile
}

PIERRE-LOUIS MAYAUX

CIRAD (UMR G-EAU) / EGE Rabat

\begin{abstract}
RESUMEN
El process tracing es un método para arribar a inferencias causales sólidas. Preocupados por la fragmentación creciente de la literatura en torno a variantes de process tracing, ponemos de relieve aquello que unifica el método: la reconstitución, desde distintas entradas, de una narrativa plausible y persuasiva para explicar resultados de interés. Nuestro argumento se construye a partir de la presentación de dos procesos de investigación, seleccionados por haberse iniciado desde dos entradas distintas: inductiva en un caso - por la novedad del fenómeno-, deductiva en el otro - por la existencia de teorías previas-. Mostramos cómo hacer process tracing rigurosamente, alternando momentos inductivos y deductivos según la entrada adoptada, y destacamos que estos estudios convergen en la producción de una narrativa que articula hipótesis y mecanismos causales para explicar los resultados de interés.
\end{abstract}

Palabras clave: metodología cualitativa, process tracing, inferencia causal, inducción, deducción

\begin{abstract}
Process tracing is a method to obtain solid causal inferences. Concerned by the fragmentation of the growing literature distinguishing different types of process tracing, we highlight the elements that unite this method: the construction, through different routes, of narratives that provide plausible, persuasive explanations of the outcomes of interest. Our argument draws on the comparison of two studies with different entry points to the research process: the first one starts inductively due to the novelty of the outcome of interest, while the second study starts deductively since previous theories were available. We show how to conduct process-tracing analysis rigorously and highlight that these studies converge to produce narratives structured around hypotheses and causal mechanisms that explain the outcomes.
\end{abstract}

Key words: qualitative methodology, process tracing, causal inference, induction, deduction 


\section{INTRODUCCIÓN}

Hasta tiempos recientes, la política comparada de metodología cualitativa se apoyaba, principalmente, en los llamados métodos de Mill de la semejanza y la diferencia. Sin embargo, asistimos a un cambio de paradigma: hoy el process tracing ocupa un lugar protagónico entre las herramientas analíticas para arribar a inferencias causales en ciencia política. A juzgar por el enorme impacto que estas nuevas reflexiones han tenido - no solo ya en los estudios de la frontera internacional de métodos mixtos (Humphreys y Jacobs 2015), sino también entre los "experimentalistas" (por ejemplo, Dunning 2012) y los politólogos formalistas (Lorentzen, Fravel, y Paine 2017, entre otros)-, es probable que en los próximos años ocurra una migración cada vez más masiva hacia el process tracing (o rastreo sistemático del proceso causal dentro de un mismo caso). El cambio de época en la metodología de la política comparada nos obliga, entonces, a profundizar nuestras reflexiones para contribuir con mayor precisión al camino ya iniciado. El presente artículo, uno de los primeros sobre el tema en lengua castellana, pone el foco en las prácticas "reales" de investigación, para aportar desde allí a la efervescente y controvertida discusión metodológica sobre process tracing.

En el pasado solíamos decir a nuestros alumnos, en las materias de métodos, que podíamos explicar fenómenos de interés mediante un diseño de investigación milliano, que contrastara "pocos" casos —típicamente, entre dos y seis- permitiendo así "controlar" o "mantener constantes" variables que adoptaban un valor semejante entre los casos, a fin de poner nuestro foco analítico y empírico en aquellas en las que los casos diferían (Przeworski y Teune 1970; Sartori 1970). ${ }^{1}$ En el presente, en cambio, observamos aquellas enseñanzas con un sano escepticismo, a partir del cual formulamos alternativas pragmáticas. Por un lado, la riqueza del método comparado continúa siendo muy significativa: la comparación de un número acotado de casos es clave para formular y refinar nuestras hipótesis, mejorar el trabajo de conceptualización y medición, y prevenir los sesgos que surgen al omitir variables relevantes. Por otro, tal como lo señala el nuevo canon metodológico dominante (Brady y Collier 2010), ya no consideramos que el método comparado de $n$ pequeño alcanza, en sí mismo, para el ejercicio de inferencia causal. Por su naturaleza, se trata de una técnica correlacional que solo retóricamente puede "controlar" los efectos de las potenciales variables independientes sobre la dependiente. ${ }^{2}$ En este artículo partimos de la premisa de que el trabajo de inferencia causal en política comparada no recae principalmente en la comparación entre los casos de estudio sino en el análisis intra-caso (Hall 2003; Collier 2011). Atendiendo y desafiando las críticas de muchos colegas que sospechan que el process tracing no es más que viejo vino en nuevas botellas, damos aquí un nuevo paso en esta

Vale aclarar que John Stuart Mill no consideraba que este método fuera aplicable a las ciencias sociales. El debate, por supuesto, no está saldado. Ver, en este sentido, Bennett y Checkel (2015: 29). 
empresa colectiva hacia la construcción de herramientas cualitativas sólidas para la inferencia causal.

Nuestro artículo elabora una visión propia que se distingue de la literatura sobre process tracing en dos importantes sentidos. Primero, nos diferenciamos de la tendencia general a dividir los así llamados "tipos" inductivos e hipotéticodeductivos de process tracing (Beach y Pedersen 2013; Trampusch y Palier 2016). Los estudios del primer tipo, iniciados sin hipótesis explicativa estructurante, comenzarían por un rastreo y una selección de los eventos claves de un proceso histórico que el investigador ensamblaría luego para formar un relato coherente y articulado mediante un conjunto de mecanismos causales interconectados. Cuando resultan efectivos, estos enfoques inductivos permitirían la formulación de nuevas hipótesis generales, o bien de nuevas interpretaciones de un proceso singular. Las variedades deductivas de process tracing, en cambio, se aplicarían para evaluar la pertinencia de hipótesis ya estructuradas. En esta perspectiva, se trataría de verificar el rol ejercido por los mecanismos causales deducidos de una hipótesis particular (o bien de varias hipótesis en competencia) en una secuencia de eventos históricos. Criticando esta visión dicotómica tan difundida en las corrientes dominantes de la literatura actual, afirmamos que no existen diferencias metodológicas fundamentales entre estas supuestamente distintas formas de process tracing. Al contrario, lo central es que todos los caminos conducen a Roma, es decir, los análisis vía process tracing, sean iniciados inductiva o deductivamente, arriban a la inferencia causal y, finalmente, producen narrativas causales complejas. Por lo tanto, más fructífero que hablar de tipos inductivos o deductivos de process tracing, es identificar lo que llamamos entradas y momentos inductivos y deductivos en el process tracing.

Segundo, consideramos que el muy sofisticado "giro bayesiano" hoy en curso (ver, especialmente, Fairfield y Charman 2015; Humphreys y Jacobs 2015), sin duda representa un avance, pero también trae consigo riesgos y debilidades. Por un lado, los bayesianos nos están permitiendo ganar claridad respecto de la arquitectura lógica del process tracing y, de este modo, disciplinar nuestro trabajo de inferencia causal (Bennett 2015). Sin embargo, a nuestro juicio, esta innovación tiene el efecto de desalentar lo que llamamos "las entradas inductivas al process tracing" y, así, desproveer a la disciplina de una herramienta que no por "vieja" es hoy menos valiosa en el intento de explicar los problemas relevantes dentro del complejo mundo de la política real. ${ }^{3}$ En línea con lo planteado por Yom (2015) en una discusión más amplia, sostenemos que desvalorizar el trabajo inductivo en pos de un deductivismo — en muchos casos solo retórico- tiene costos altos en materia de transparencia, ya que se abre una brecha entre lo que se dice que se hizo durante el proceso de investigación y lo que efectivamente fue realizado. Asimismo, consideramos que el enfoque bayesiano, sin dudas loable en sus objetivos, ha elegido un camino que tiende a inducir la formación 
de aquello que Sartori (1970) llamó "pensadores sobre-conscientes". El riesgo de auto-imponerse estándares con exceso de ambición y adoptar este enfoque acríticamente, es semejante al que identificara Sartori (1970: 1033, 1053): pasar a ser dominados por el método y quedar paralizados como investigadores. ${ }^{4}$

Adoptamos, entonces, una perspectiva distinta que se concentra en la práctica misma de la investigación. Nuestra contribución pragmático-incremental tiene dos ventajas: por un lado, el anclaje en los dilemas que enfrentamos los investigadores en el "mundo real" habilitará a futuros investigadores no familiarizados con este nuevo canon a comprender con mayor agilidad y efectividad el potencial de estas herramientas - así como sus principales riesgos analíticos- y a poder enseñarlas más fácilmente a los investigadores en formación y, por otro, nuestra colaboración se hace eco - y predica con el ejemplo- de un movimiento cada vez más dinámico en pos de aumentar los grados de transparencia en los procedimientos de investigación, y en maximizar el potencial de nuestros estudios de ser "replicables" por otros colegas disconformes con las explicaciones a las que arribamos (Hall 2016). Consideramos que la transparencia en nuestra disciplina se juega, en buena medida, en nuestra capacidad de echar luz sobre las prácticas reales de los investigadores en vez de ocultarlas o estigmatizarlas (Yom 2015).

A fin de elaborar el argumento para una visión metodológicamente unificada del process tracing, reconstruimos aquí dos procesos de investigación en sus distintas etapas. Reunimos las experiencias de un trabajo iniciado a partir de la exploración del material empírico y otro que partió con la revisión de hipótesis consolidadas en la literatura. El primer caso da cuenta de una investigación sobre el mecanismo causal que denomináramos policy trap, evidenciado en Argentina luego del incremento exponencial del gasto público en subsidios a los servicios públicos entre la postcrisis de 2001 y el presente. Por ser un fenómeno que no había suscitado interés en la ciencia política cuando empezamos a estudiarlo, tuvimos que adoptar una entrada inductiva, es decir, empezar la investigación por la recolección y sistematización exploratoria de datos, dando así inicio a una secuencia particular de acciones de investigación que, solo más tarde, daría paso al momento deductivo. En cambio, la aproximación al segundo caso, la movilización social de gran envergadura ocurrida en 2000 en Cochabamba (Bolivia) contra la privatización del sistema de agua de la ciudad — conocida como la "Guerra del agua"—, se realizó mediante una entrada deductiva. Esto fue posible por la existencia de teorías sobre el fenómeno del rechazo a la privatización de los servicios hídricos, así como de relatos específicos de este episodio de acción contenciosa. En la investigación sobre Bolivia, los momentos inductivos fueron posteriores a los deductivos. A pesar de esas diferencias en el punto de inicio, los resultados de investigación son esencialmente similares,

Aun los exponentes más destacados del enfoque bayesiano (Humphreys y Jacobs 2015) reconocen que el procedimiento inferencial que proponen es tan demandante que puede terminar por reducir fuertemente el alcance de su uso. 
ya que las inferencias causales construidas en estos dos trabajos se caracterizan, principalmente, por su fuerte validez interna, pero también pueden ser extendidas para dar cuenta de otras instancias de una misma clase de fenómeno (Gerring 2007). El desafío de nuestro artículo es, entonces, tanto analítico como pedagógico. Por un lado, ponemos en evidencia el modo por el cual los recorridos metodológicos conducen, desde entradas diferentes, a resultados equivalentes. Por otro, al hacer este análisis sobre los estudios, mostramos sin rodeos ni velos tecnicistas- cómo puede hacerse hoy en día investigación cualitativa rigurosa.

Primero, a partir de una discusión crítica de la literatura especializada, elaboramos un marco unificado para la realización de process tracing, independientemente del punto de entrada inductivo o deductivo. Luego, sustentamos nuestros argumentos mediante la presentación detallada de los procesos de investigación sobre policy traps y la "Guerra del agua", haciendo visible para el lector cada etapa metodológica en la reconstitución del proceso. El examen de estos dos casos nos faculta para entregar lineamientos para la realización de un buen process tracing, identificando buenas prácticas que se suman a las que ya destacó la literatura. La conclusión explora el impacto que pueden tener los hallazgos de este artículo en las nuevas agendas empíricas y metodológicas.

\section{MÁS ALLÁ DE LOS TIPOS DE PROCESS TRACING: UNA PRÁCTICA COMÚN DE ARTICULACIÓN DE MOMENTOS DEDUCTIVOS E INDUCTIVOS}

Proponemos una visión unificada de las prácticas del process tracing, en la cual coexisten, por una parte, momentos con predominio deductivo y, por otra, ajustes sucesivos entre inducción y deducción. La tendencia en la literatura especializada a diferenciar entre tipos inductivos e hipotético-deductivos en el process tracing (Beach y Pedersen 2013; Bennett y Checkel 2015; Trampusch y Palier 2016), a nuestro juicio, no da cuenta de las prácticas reales de este método. Al contrario, exacerba diferencias y, por lo tanto, impide percibir aquello que estos tipos ideales tienen en común.

Hoy existe amplio acuerdo sobre ciertos aspectos comunes en las investigaciones que se construyen vía process tracing, tales como la referencia sistemática a los mecanismos causales o el carácter complejo de la inferencia causal a la que se busca llegar. Pero, al mismo tiempo, gran parte de los autores insisten en la existencia de variaciones significativas entre distintos trabajos de process tracing. Así, Bennett y Checkel (2015) distinguen los trabajos de process tracing de acuerdo a si prueban una teoría o a si construyen una nueva (theory-testing o theory-building). Sostenemos que estas diferenciaciones generan riesgos de fragmentación que podrían terminar por debilitar la difusión del método. Sobre todo, estas distinciones impiden ver los elementos comunes en prácticas que 
convergen, más allá de entradas diferentes. En consecuencia, nos parece más importante insistir sobre la heurística común de este método fundado sobre la articulación entre eventos, variables y mecanismos causales.

Esta unidad en las prácticas no es ajena para algunos de estos autores. Por ejemplo, los propios Bennett y Checkel (2015: 7) proponen una definición unificada del process tracing como "el análisis de la evidencia sobre los procesos, secuencias y coyunturas de eventos dentro de un caso con el propósito de desarrollar o testear hipótesis sobre los mecanismos causales que podrían explicar causalmente el caso". Esta definición se refiere a lo que luego llaman dos "lados" (sides): el inductivo se asocia con la generación de hipótesis y el deductivo con su testeo. Sin embargo, esta asociación entre, por un lado, dos procedimientos metodológicos como la inducción y la deducción - es decir, en el primero partir de la evidencia empírica para avanzar hacia la teoría o, en el segundo, iniciar la investigación desde la teoría existente, para luego confrontarse con la empiria (Ragin 2007) - y por el otro lado, dos objetivos de investigación - construir o testear teoría - no refleja la práctica concreta de los cientistas políticos (Yom 2015). Así, esta separación entre tipos resulta frágil y relativamente arbitraria. Incluso es posible cuestionar su pertinencia.

Nuestro escepticismo en cuanto a estas clasificaciones se aplica también a la reflexión tipológica de Beach y Pedersen (2013), quienes separan entre tres "variantes" y "usos" del process tracing. A las tradicionales variantes de testeo y construcción de teoría, suman una tercera, explaining-outcome process tracing, dedicada a explicar un resultado político particular (Beach y Pedersen 2013: 12). Esta abre espacio para dos "caminos" (paths) para la investigación, inductivo y deductivo. Sin embargo, los dos autores circunscriben esta posibilidad a una versión particular de process tracing, reservada, según ellos, a un público de profesionales preocupados por explicaciones exclusivamente empíricas y carentes de ambiciones teóricas. A nuestro juicio, tal restricción no tiene fundamentos metodológicos.

Nosotros sostenemos, en cambio, que todo process tracing se compone de elementos fundamentales que son la entrada y los posteriores distintos momentos. Tanto la entrada - el inicio del proceso de investigación - como los momentos - las distintas etapas de su desarrollo- pueden ser inductivos o deductivos. Así, luego de iteraciones entre momentos inductivos y deductivos, el proceso de investigación converge hacia la explicación del resultado de interés. Debemos, por lo tanto, abandonar la ficción que sostiene que todo proyecto de investigación estaría sobre-determinado por un supuesto carácter deductivo $o$ inductivo, que se elige en el momento t 0 , y daría origen a senderos que se bifurcan en $t[1, \ldots, n]$. Al trascender la dicotomía entre tipos de process tracing, por un lado, y al alertar sobre el riesgo de desaliento que surgiría si concibiéramos al deductivismo del giro bayesiano como único canon capaz de guiar el process tracing riguroso, por el otro, nuestro aporte se centra en las prácticas. La propuesta parte de la convicción de que, para proteger el principio de transparencia que 
regula el trabajo científico, es necesario reducir la incongruencia hoy existente entre lo que los investigadores explicitan y sus prácticas implícitas (Yom 2015).

Enfocarnos en las prácticas nos permite admitir, sin rodeos, aquello que hoy suele estar velado: muchos investigadores iniciamos investigaciones simplemente porque un fenómeno empírico nos parece intrínsecamente interesante, de alta relevancia, y que por esta precisa razón debe ser estudiado con urgencia, aunque no exista ni siquiera la información más básica para sistematizar la empiria ni certeza sobre qué teoría existente podría contribuir a explicarlo. En otras palabras, como admiten incluso los más deductivistas (Bennett y Checkel 2015: 271), muchos process tracers somos, ante todo, investigadores motivados, orientados o incluso "obligados" por los problemas del mundo real. ${ }^{5}$ A diferencia de la tendencia dominante en la literatura, sostenemos que el estudio de estos fenómenos que nos desvelan no tiene nada en sí mismo de a-teórico... pero sí puede determinar que la entrada hipotética-deductiva no pueda adoptarse al inicio y que el razonamiento deductivo solo aparezca, con toda su fuerza, en un segundo o tercer momento de la investigación. Por estas razones, en este artículo hablamos de entradas y momentos deductivos e inductivos, no de tipos o enfoques. Este lenguaje nos permite descubrir, con mayor precisión, lo que efectivamente sucede en la práctica de la investigación vía process tracing, motivando nuevas investigaciones que ganen en conciencia, sin por ello quedar atrapadas en abstracciones rígidas que obstaculicen su avance.

Luego de haber mostrado la irrelevancia de separar el process tracing en tipos, campos o variantes, queda entonces por determinar cuándo privilegiar una $u$ otra entrada, y cuándo concluir el proceso de iteración. La decisión respecto de si el recorrido inicial es más inductivo o deductivo, generalmente está constreñida por el estado de la literatura sobre la pregunta de investigación y el caso trabajado. Por un lado, la entrada inductiva implica pasar primero por los hechos, construyendo una primera narración no "causalizada", aunque guiada por intuiciones teóricas. Se elige este camino cuando existe poco o ningún conocimiento sistemático sobre el fenómeno de interés. En cambio, la entrada por la deducción comienza por la (re)construcción y la prueba de las hipótesis, lo cual supone que estas preexisten al proyecto de investigación. Así, las hipótesis pueden nacer de la literatura y de la observación empírica. En cualquier caso, la creatividad del investigador es imprescindible para, siguiendo la recomendación de Bennett y Checkel (2015: 18), desplegar la red lo más ampliamente posible en la "pesca" de posibles explicaciones alternativas del fenómeno en estudio.

Entonces, más allá de la entrada, todo process tracing cuenta con momentos con predominancia deductiva fundada sobre las "implicancias observables" de las hipótesis formuladas. ${ }^{6}$ Se trata de deducir, a priori, ciertas implicancias empíricas

“La realidad obliga.” Memorable primera oración de un trabajo de O’Donnell (1978: 1).

Sobre implicancias observables, ver King, Keohane, y Verba (1994: 28-9) y Brady y Collier (2010). 
de un mecanismo, antes de verificar si estas efectivamente se observan en el registro empírico. A los momentos deductivos suceden momentos inductivos, de elaboración teórica a partir de la confrontación con la materia empírica. ${ }^{7}$ Así, las hipótesis son ajustadas mediante un proceso iterativo en el cual una sucesión de relatos, todavía insatisfactorios, se aproximan gradualmente hacia una narrativa finalmente juzgada como "plausible" y "persuasiva" (Yom 2015: 626).

El proceso de investigación llega a su fin cuando se cumplen dos condiciones. Por parte del investigador, se estima haber alcanzado el punto de saturación. Este término, de amplio uso en la metodología cualitativa, se refiere a la baja probabilidad de obtener mayor información relevante si prosiguiera la recolección de datos, es decir, a los rendimientos decrecientes del trabajo de campo. Saturación también se refiere a la elaboración teórica, en el sentido de que el investigador considera haber explicado el caso de interés de manera plausible. La segunda condición, asociada al carácter persuasivo de la explicación, es la validación por pares. Esta apreciación intersubjetiva puede ocurrir en distintas instancias (comité de tesis, árbitros de revistas, entre otras), según el contexto institucional en el cual se desarrolla la investigación.

La Figura 1 da cuenta de nuestra propuesta de un marco unificado para la práctica del process tracing, donde, luego de iniciar la investigación por entradas diferentes, el desarrollo sigue un curso de progresión y de ajuste equivalente, para llegar a un resultado del mismo orden.

En sociología se ha propuesto el término de "abducción" para dar cuenta de este proceso de teorización a partir de evidencia empírica sorprendente (Timmerman y Savory 2012). Sin embargo, nos parece más fructífero destacar la articulación entre las operaciones tradicionales y bien conocidas de inducción y deducción, que proponer una categoría nueva que dificulta la acumulación de conocimiento en el campo de la metodología. 
Figura 1. El process tracing en la práctica: diferentes entradas para generar inferencias causales

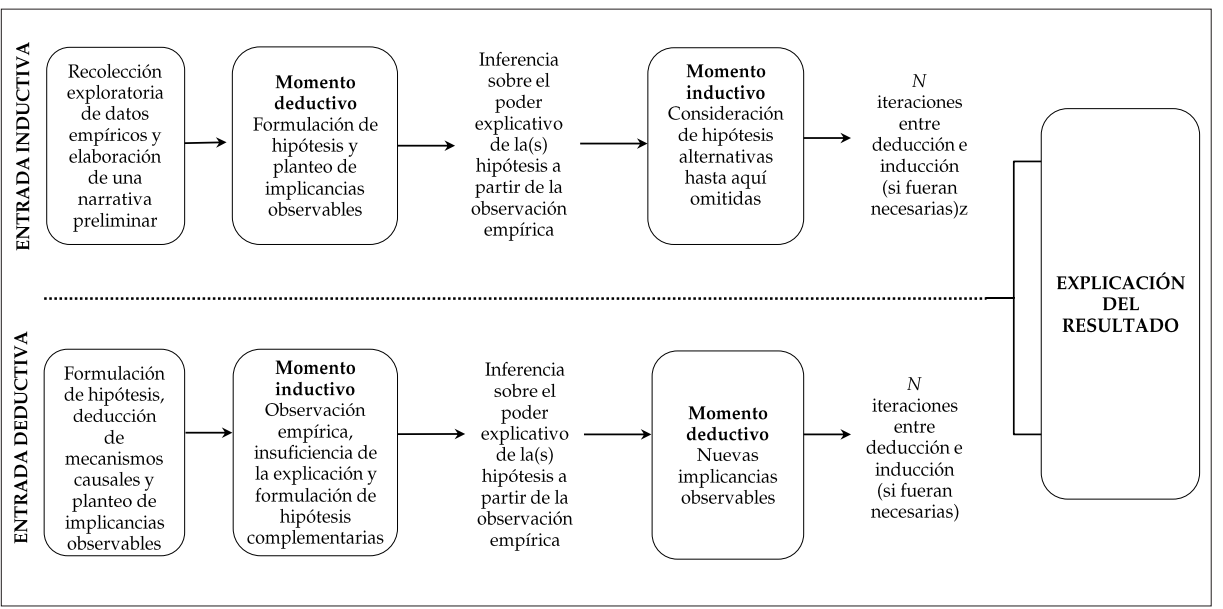

Fuente: elaboración propia

El process tracing aumenta así la plausibilidad del relato final, a partir de la interacción entre la generación hipotética de implicancias observables y las evidencias que las respaldan o refutan, reiterada tantas veces como sea necesario. El corolario de estos momentos deductivos es que el investigador es invitado a poner claramente a disposición del lector sus hipótesis en relación a las observaciones esperadas. Así, dotados de este concepto de process tracing como método articulador de la inducción y la deducción a través de ajustes sucesivos, vamos a proceder a la presentación de dos procesos de investigación para mostrar prácticamente que las similitudes esenciales prevalecen sobre las diferencias accesorias.

\section{ENTRADA INDUCTIVA AL PROCESS TRACING. SUBSIDIOS DE AMPLIO ESPECTRO EN SERVICIOS PÚBLICOS EN LA ARGENTINA (2003-PRESENTE)}

En esta sección ilustramos la entrada inductiva al process tracing, analizando una investigación sobre los subsidios de amplio espectro en servicios públicos en la Argentina de principios del siglo xxI (Bril-Mascarenhas y Post 2014, 2015). En apenas una década, los subsidios que el Estado nacional argentino creó en la poscrisis de 2001 — para contener los aumentos tarifarios en los servicios de gas, electricidad, agua y transporte urbano- pasaron de ser un paliativo con muy bajo costo fiscal, a la principal fuente del actual alto déficit presupuestario. Al momento de escribir estas líneas, los subsidios en servicios públicos ocupan un lugar central en la agenda pública en la Argentina, debido a la dificultad que el gobierno de Mauricio Macri tiene para, sin afectar en exceso sus chances 
de reelección, desmontar lo que Bril-Mascarenhas y Post (2015) denominaron la policy trap de los subsidios. Sin embargo, cuando en 2010 se inició esta investigación, no había ningún análisis sistemático sobre esta área de política pública, quizá porque jamás se sospechaba que este tema pasaría —en pocos años- de ser una cuestión muy técnica y de baja visibilidad pública, a uno de los ejes del conflicto político en la Argentina post-kirchnerista.

Aquella investigación estuvo, entonces, motivada por genuina curiosidad empírica y teórica. Por un lado, los autores estaban impactados por una realidad cuya importancia pocos habían advertido hasta entonces: apenas siete años después de haber sido instituidos, los subsidios en servicios públicos absorbían el $10 \%$ del presupuesto. Asimismo, mostraban una dinámica de crecimiento vertiginoso y casi automático. Paradójicamente, no obstante, los gobiernos kirchneristas obtenían un rédito político-electoral significativamente menor de estos subsidios que de otras políticas públicas. Por otro lado, la perplejidad teórica surgía de notar que la ciencia política internacional —abocada al estudio de las transferencias más clásicas del estado de bienestar - había hecho poquísimo por explicar el origen, enraizamiento y enorme crecimiento de los subsidios de amplio espectro al consumo. Debido a ello, este terreno empírico quedaba casi exclusivamente en manos de los economistas.

De este modo, la ausencia de desarrollo teórico previo obligaba a la entrada inductiva. Esto implicó un significativo esfuerzo de recolección de estadísticas sobre el costo fiscal y la distribución territorial de los subsidios en servicios públicos. En simultáneo, los investigadores, desprovistos aún de hipótesis que ameritaran ser testeadas, se dedicaron a hacer trabajo de archivo a fin de reconstruir la trayectoria de esta política pública. En la identificación y sistematización de los hitos regulatorios o nodos de decisión gubernamental durante las administraciones de Néstor y Cristina Kirchner (2003-2015), fue clave evitar el sesgo de selección hacia hitos regulatorios efectivamente ocurridos. Tan importantes como ellos, son lo que aquí podríamos denominar "nodos de no-decisión" - es decir, situaciones en las cuales el poder ejecutivo desistió de tomar un curso de acción que estaba considerando o bien lo tomó para luego dar marcha atrás de inmediato-. La exhaustiva identificación, tanto de los hitos regulatorios como de los nodos de no-decisión relevantes, es fundamental para un ejercicio exitoso de inferencia causal vía process tracing.

Concluida la sistematización de datos secundarios, los investigadores escribieron una primera narrativa del proceso de adopción y crecimiento de los subsidios en el período bajo estudio. Este relato inicial carecía aún de cualquier impronta explicativa: dado el subdesarrollo del estudio sobre el tema, en esta etapa habría sido muy difícil ir más allá de la descripción. El relato estaba, por lo tanto, desprovisto de macro-causas, mecanismos causales o evaluaciones de hipótesis alternativas. Con este texto inicial, se procedió al trabajo de campo exploratorio, cuyo principal insumo fue una serie de entrevistas con una muestra 
no probabilística ${ }^{8}$ de altos decisores en el Estado nacional, los sindicatos y las empresas de dos sectores subsidiados — distribución de gas y transporte público urbano (colectivos) - . El objetivo aquí era adquirir una profundidad que, en los hechos, es imposible de obtener vía datos secundarios. Esta primera ronda de entrevistas buscaba, entonces, aproximarse hacia posibles microfundamentos del comportamiento de los actores potencialmente relevantes en esta arena de políticas.

Se elaboró, entonces, una descripción cuantitativa de la evolución de la política pública de subsidios de amplio espectro en servicios públicos en la Argentina de la postcrisis de 2001 y se arribó por la entrada inductiva a una narrativa causal preliminar. La estadística descriptiva indicaba que hubo una significativa variación intra-caso en Argentina a través del tiempo. Hacia 2010, los subsidios del Estado nacional para contener aumentos de las tarifas de los servicios públicos habían crecido hasta alcanzar los 7.500 millones de dólares anuales. Además, se distribuían de manera significativamente desproporcional en el territorio argentino, con un gran sesgo hacia el Gran Buenos Aires (GBA). La narrativa causal preliminar, por su parte, sugería que el enraizamiento y exponencial crecimiento de los subsidios de amplio espectro en distribución de gas y transporte en colectivo podrían rastrearse en la interacción de dos factores: las presiones de precios (por ejemplo, el aumento del costo del gas importado) y los desincentivos políticos que constreñían potenciales iniciativas para desmantelar esta política pública (tales como el temor a sufrir un revés electoral en distritos altamente subsidiados).

Los autores dieron paso al momento deductivo de la investigación. Esta etapa, como veremos, no estuvo exenta de nuevas iteraciones inductivas. Tal como lo estilizáramos en el marco analítico, en la investigación sobre subsidios en la Argentina los autores formularon implicancias observables a partir de cuerpos teóricos considerados relevantes. Dado que el gasto público en subsidios se concentraba en el GBA —-donde reside un tercio de la población argentina-, se buscó, en primer lugar, una brújula analítica en la literatura sobre el "sesgo urbano" de las políticas públicas (ver, entre otros, Bates 1981). De allí, y de las proposiciones derivadas de las literaturas sobre blame avoidance - es decir, la dinámica de la "evasión de la culpa política" (ver Weaver 1986)—, BrilMascarenhas y Post (2015) se propusieron observar si un mecanismo causal relevante en la explicación del enraizamiento de los subsidios en Argentina era la predominancia de una estrategia de elusión de la culpa política por parte de las administraciones de Néstor y Cristina Kirchner. En línea con las teorías que muestran que la ciudadanía tiende a ser más sensible ante las pérdidas que ante las ganancias o los beneficios que ya recibe (Weyland 1996), Bril-Mascarenhas y Post (2015) analizaron en qué medida el proceso estuvo marcado por el

8 Sobre process tracing y entrevistas a élites con muestras no probabilísticas ver Tansey (2007), quien argumenta que el muestreo aleatorio va en contra de la lógica del process tracing, en tanto genera un alto riesgo de excluir de la muestra a actores claves en los procesos estudiados. 
temor de los presidentes entre 2003 y 2015 por enfrentar reveses electorales y/o el surgimiento de protestas con alto impacto político en la principal área metropolitana de Argentina.

Triangulando evidencia surgida de una nueva ronda de entrevistas confidenciales con actores claves en los procesos decisorios de esta política pública con trabajo de archivo y encuestas de opinión pública, Bril-Mascarenhas y Post (2015) infirieron que, efectivamente, la persistencia de esta política pública en el caso de Argentina había obedecido principalmente al temor de los líderes políticos a autoinfligirse un revés electoral. Toda la evidencia recabada apuntaba en la misma dirección: desde el poder ejecutivo se percibía que el recorte de los subsidios y el consecuente drástico aumento de las tarifas de servicios públicos tendrían el efecto de volcar a la población del GBA y, con ella, a la opinión pública nacional, en contra de la administración kirchnerista. Los investigadores detectaron, asimismo, un efecto de interacción entre variables políticas y económicas: las presiones de precios aumentaron con el paso del tiempo y el potencial (ya, de por sí, alto) costo político de la salida del esquema de subsidios. Se observó, por lo tanto, que, si bien la motivación inicial que había dado origen a los subsidios de amplio espectro en la Argentina fue contener el impacto del shock económico de 2002 sobre el conjunto de la población, el enraizamiento de esta política pública obedecía, principalmente, a un mecanismo causal específico: la estrategia de blame avoidance escogida por el ejecutivo.

Mientras se avanzaba en el estudio del caso de Argentina, los investigadores sistematizaron datos sobre 29 países en desarrollo que indicaron que los subsidios al consumo constituyen un importante dispositivo de protección social: en muchos países el presupuesto en subsidios superaba el gasto público en salud y educación. Esta observación inductiva a partir de un $n$ relativamente grande, junto con la recomendación de un réferi anónimo, motivó la consideración del cuerpo teórico sobre el estado de bienestar (EB), a fin de derivar desde allí implicancias observables para el análisis del caso argentino. La literatura argumenta que los programas del EB generan grupos organizados y concentrados de beneficiarios que tienen un claro interés por $-\mathrm{y}$, a menudo, la capacidad de- evitar su desmantelamiento. Si estas teorías gozaran de alto poder explicativo para dar cuenta de la trayectoria bajo análisis, un factor clave, conducente al crecimiento y el enraizamiento de los subsidios en Argentina, debía ser la presión ejercida por grupos organizados de beneficiarios, como los sindicatos y las cámaras empresariales de los sectores subsidiados.

Bril-Mascarenhas y Post (2015) utilizaron un diseño de investigación que yuxtaponía una comparación intersectorial $(n=2)$ y el rastreo sistemático del proceso causal dentro de cada sector. El análisis comparado de los servicios de distribución de gas y de transporte público mostró que los mecanismos causales resaltados por las teorías sobre el EB no eran necesarios para el crecimiento y el enraizamiento de los subsidios: si bien en el sector transporte, tanto los sindicatos como las cámaras empresariales estaban sólidamente organizados, en el sector de distribución de gas los subsidios crecieron y se enraizaron aun en 
la ausencia de presiones por parte de grupos de interés organizados. El hecho de que las implicancias de las teorías del EB no se observaran en el registro empírico fortaleció el argumento de los autores de que el aumento del costo de los insumos y el temor político ante potenciales reveses electorales operaron como condiciones suficientes para el crecimiento y el enraizamiento de los subsidios de amplio espectro en la Argentina entre 2003 y 2015.

En síntesis, el artículo de Bril-Mascarenhas y Post (2015) ilustra cómo, desde una entrada inductiva, los autores arribaron a una narrativa causal (Figura 2). En el camino, la investigación pasó por una serie de iteraciones que supusieron tomar perspectiva del caso, volver a la teoría y, durante momentos deductivos, derivar un conjunto de proposiciones a ser contrastadas en nuevas rondas de trabajo empírico. Este artículo muestra, asimismo, que, para dar por concluidas las iteraciones entre inducción y deducción, es importante indagar sobre la presencia o ausencia de efectos de interacción entre las variables explicativas. En línea con nuestro marco, el producto final de Bril-Mascarenhas y Post (2015) de ningún modo restituye la riqueza histórica del proceso estudiado: el trabajo de process tracing, lejos de buscar ese tipo de descripción densa, utiliza evidencia de un mismo caso para lograr el objetivo mayor de hacer inferencias sobre las explicaciones causales de dicho caso (Bennett y Checkel 2015: 4-8).

Figura 2. Ilustración de la entrada inductiva al process tracing: formación de una policy trap en la Argentina

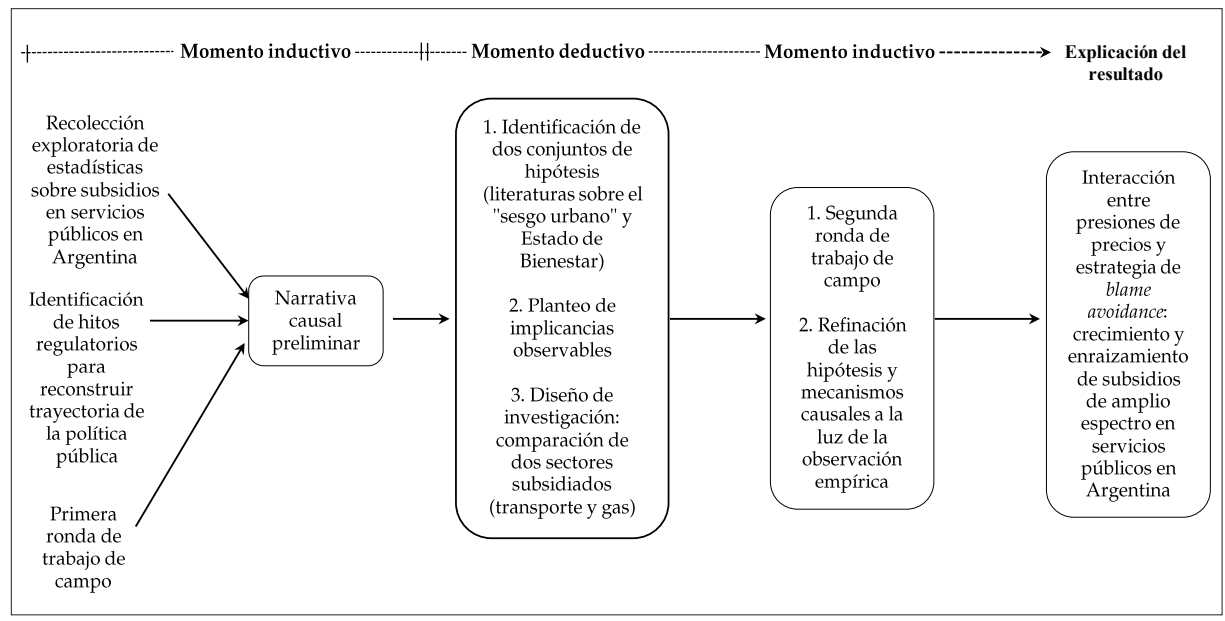

Fuente: elaboración propia

Como bonus analítico, el estudio intra-caso de los subsidios en Argentina fue el puntapié inicial para observar un gran conjunto de casos de países en desarrollo y para elaborar una lógica sobre lo que Bril-Mascarenhas y Post (2015) denominaron policy traps. Cerrando el círculo —o, más bien, volviendo a abrirlo- las proposiciones que estos autores elaboraron sobre la lógica política 
de las policy traps invitan a encarar nuevos trabajos de process tracing que se inicien por la entrada deductiva, derivando implicancias observables — desde el marco analítico de las policy traps - para evaluar si esta lógica explica mejor o peor que otras teorías alternativas ciertos procesos de políticas públicas (tales como la persistencia de ciertos regímenes de tipo de cambio) en múltiples países y períodos históricos.

\section{ENTRADA HIPOTÉTICO-DEDUCTIVA AL PROCESS TRACING. LA “GUERRA DEL AGUA" EN BOLIVIA COMO UN CASO DE MOVILIZACIÓN SOCIAL EXCEPCIONAL}

En septiembre de 1999 se firmó un contrato de concesión por cuarenta años del servicio de agua potable y saneamiento de la ciudad de Cochabamba, otorgada al operador Aguas del Tunari, cuyo capital mayoritario pertenecía al grupo estadounidense Bechtel. Esta reforma en el manejo del recurso hídrico en Cochabamba contaba con un apoyo transversal y multinivel, incluyendo al alcalde, autoridades nacionales y empresarios locales. Sin embargo, menos de un año después, en abril de 2000, después de una serie de protestas, el gobierno resolvió rescindir el contrato. Así llegó a término este episodio emblemático de la vida política boliviana conocido como la "Guerra del agua". ¿Por qué y cómo se conformó una movilización social tan potente que logró sobreponerse a esta coalición de las élites para, finalmente, forzar la anulación del contrato? Para responder esta pregunta se recurrió a un process tracing con entrada deductiva, del cual damos cuenta aquí.

El punto de partida para la reflexión deductiva fue teórico y radicó en dos literaturas aparentemente opuestas: las teorías neoclásicas sobre el proceso de privatización, por un lado y, por otro, análisis críticos de las consecuencias de la incorporación de actores privados para la gestión de un recurso que algunos consideran un bien común. Ambas corrientes coinciden en poner el foco en las alzas de tarifa que, generalmente, acompañan los procesos de privatización para explicar su destino final. Diversos relatos sobre la "Guerra del agua" también señalaban el alza tarifaria como un posible desencadenante de la movilización social. La lectura de estas teorías condujo a la formulación deductiva de la siguiente hipótesis: las alzas tarifarias propias de estos procesos habían provocado un levantamiento de la población pobre de Cochabamba.

Desde el punto de vista del proceso de investigación, la pregunta fundamental era determinar si esta hipótesis, en caso de ser comprobada, podría ser considerada plausible y convincente para explicar el resultado. Un paso inicial consistió en elaborar, deductivamente, un mecanismo causal, del cual la variable alza tarifaria fuera el detonante. Para la construcción ex ante de una hipótesis "mecanísmica" (mechanismic) (McAdam, Tarrow, y Tilly 2008), Mayaux (2012) se apoyó en trabajos ya clásicos de la sociología de las movilizaciones sociales. Hipotetizó inicialmente dos mecanismos mediante los cuales el 
encarecimiento del servicio produciría un movimiento de la envergadura de la "Guerra del agua". Primero, se buscó establecer la presencia del mecanismo de "enmarcamiento" (framing). Las implicancias observables deducidas serían, entonces, acciones de grupos organizados que relacionaran las alzas con la privatización, contribuyendo a que los aumentos tarifarios no aparecieran como algo menor o aislado, sino como parte de una transformación amplia. El segundo mecanismo considerado ex ante fue la polarización que se gatillaría por un rechazo de la coalición dominante por revisar estos aumentos, lo que tendría como efecto una amplificación de la contestación, contribuyendo así a que el rechazo escalara de un problema puntual con los precios a un repudio global de la privatización.La Figura 3 representa ambas hipótesis "mecanísmicas".

Figura 3. Bolivia. Mecanismos elaborados deductivamente

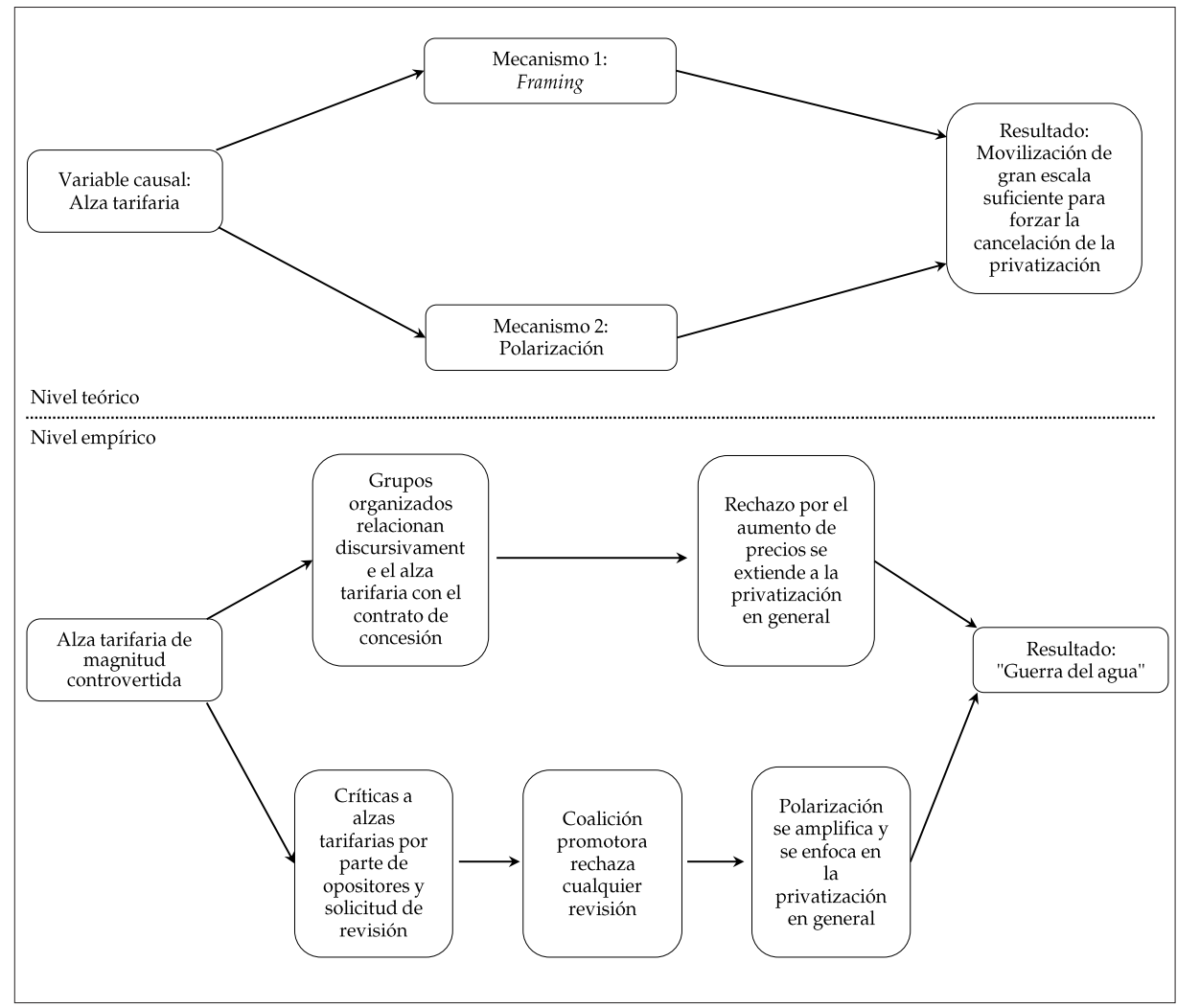

Fuente: elaboración propia

La Figura 3, inspirada en Beach y Pedersen (2013), indica — en la parte de arriba (nivel teórico) - los posibles mecanismos elaborados a partir de la teoría de la movilización social. En la parte baja (nivel empírico), aparece el encadenamiento 
causal asociado a cada mecanismo, donde los distintos eslabones son implicancias observables, cuya presencia o ausencia debe ser constatada en el registro empírico.

La etapa siguiente del proceso investigativo consistió en confrontar estas hipótesis con la evidencia. En primer lugar, la evidencia recolectada permitió mostrar que el mecanismo de framing había operado. En efecto, el incremento de un $35 \%$ promedio de las tarifas fue activamente interpretado por un grupo clave de la oposición, la Coordinadora por la Defensa del Agua y de la Vida (Coordinadora), como consecuencia directa de la privatización. Luego aparecieron varios elementos discursivos que conectaban directamente el alza con un cuestionamiento más profundo, que incluía elementos tanto de lo "nacionalpopular" como de reivindicaciones indígenas. En cambio, el mecanismo de polarización elaborado deductivamente no resistió la confrontación con la realidad empírica. Así, no se produjo la polarización hipotetizada, al menos del lado de la coalición dominante. En consecuencia, la reconstitución precisa de la dinámica de la movilización mostró el carácter incompleto del mecanismo elaborado deductivamente. Se infirió que sería erróneo atribuir al incremento tarifario un carácter detonante exclusivo y se volvió necesario identificar variables y mecanismos complementarios.

En esta fase ya avanzada de la investigación fue posible llevar a cabo dicha tarea de manera inductiva a partir de la evidencia ya recolectada. Así, en línea con la crítica a la oposición dicotómica entre tipos de process tracing, se articularon momentos deductivos e inductivos para producir una explicación plausible. Durante la primera etapa, había resultado llamativa la presencia conjunta en la Coordinadora de usuarios urbanos y rurales. El conocimiento histórico del caso hacía parecer sorprendente que estos grupos pudieran sortear problemas de coordinación para actuar colectivamente, ya que había habido múltiples conflictos a lo largo de décadas anteriores entre actores asociados a distintos tipos de uso del agua. Esta coalición inédita podía ser interpretada como el producto de un mecanismo causal de brokerage, complementario al framing ya comprobado.

Para sustentar esta hipótesis elaborada inductivamente faltaba encontrar la variable causante del proceso. El análisis de las entrevistas reveló que un motivo para la movilización había sido el riesgo de expropiación, que no tenía sus raíces en el contrato de concesión per se, sino en la ley nacional del agua promulgada en noviembre de 1999 (Congreso Nacional de Bolivia, 1999). La ley entregaba a los concesionarios un derecho de propiedad exclusivo sobre todo el recurso hídrico en su definida zona de explotación. Esto constituía una amenaza directa para las cooperativas y "comités de agua" que habían constituido pequeñas redes de abastecimiento. Profundizando este razonamiento, fue posible reconstituir el proceso de brokerage entre dirigentes rurales urbanos, para finalmente establecer la Coordinadora. Así, a la oposición articulada en torno de los aumentos tarifarios, luego "enmarcados" como parte de una crítica general a la privatización, le había antecedido la construcción de una 
compleja estructura basada en la convergencia inédita de actores, producto del mecanismo de brokerage.

Este mecanismo se suma al anteriormente comprobado de framing, gatillado por el aumento tarifario. En este punto del trabajo de process tracing correspondía preguntarse si se había llegado a una explicación plausible y convincente del resultado. En este caso, el investigador evaluó que existía todavía un residuo significativo por dilucidar. En efecto, quedaba por explicar la durabilidad y fuerza de la movilización, a pesar de la disposición del gobierno a revisar las tarifas, así como de una fuerte represión y estrategias de cooptación. El brokerage, junto con un framing de la problemática específica, ¿habría bastado para organizar en tres oportunidades un bloqueo completo de la ciudad? A la luz del conocimiento del investigador, tanto del caso nacional como de otras instancias de conflicto en torno a procesos de privatización, dichos factores no parecían agotar la explicación del fenómeno bajo estudio. Todavía era necesario dar un paso más en el análisis.

Así, se volvió a analizar el proceso completo. La nueva revisión de la evidencia empírica recolectada permitió conjeturar sobre la presencia de un mecanismo más profundo de "cambio de identidad" (identity shift) (Tilly y Tarrow 2007). La omnipresencia del slogan "el agua es nuestra, carajo" parecía indicar que éste podría ser un mecanismo causal relevante. Esta consigna fue clave para cimentar una identidad colectiva de desposeídos. Produjo un nuevo sentido común, compartido por todos los actores movilizados, en oposición a la élite privatizadora. La violencia de la represión contribuyó fuertemente a consolidar esta nueva identidad, en particular porque reactivó la memoria de la dictadura de Hugo Banzer (1971-1978). Esta identidad se sustentó, también, en prácticas participativas que permitieron reforzar el compromiso de los militantes y el contraste con "los otros" y, así, robustecer una identidad alternativa que fue fundamental para darle sustento a la movilización social, hasta lograr que el gobierno cancelara el contrato de concesión.

Con la elaboración inductiva de este tercer mecanismo, producto del efecto cumulativo de los otros dos constatados — framing y brokerage-, y con rasgos del que inicialmente se había deducido - polarización-, Mayaux (2012) estimó disponer de una explicación convincente de esta movilización social de excepcional envergadura. La Figura 4 sintetiza el efecto combinado de los tres mecanismos causales detectados. 
Figura 4. Narrativa causal plausible para explicar la "Guerra del agua" en Bolivia

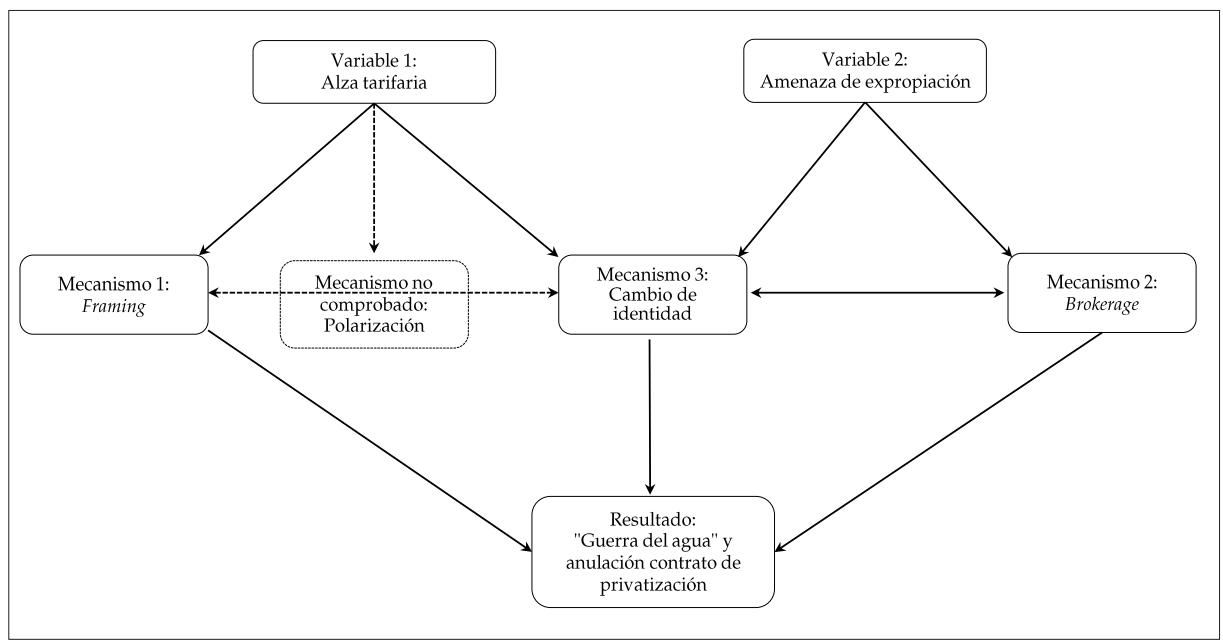

Fuente: elaboración propia

Más allá de la "Guerra del agua", la combinación verificada de mecanismos constituye un insumo para estudios sobre procesos de movilización social territorial de gran envergadura. Por ejemplo, se podría trabajar sobre las movilizaciones sociales ocurridas en el sur de Chile a principio de la década de 2010, formulando hipótesis a partir de los hallazgos de esta investigación, sea para confirmar y extender los mecanismos causales o para complementarlos con otros propios de estos casos.

\section{ALGUNAS LECCIONES Y RECOMENDACIONES PRÁCTICAS}

La presentación detallada de dos procesos de investigación permitió poner a la vista las etapas concretas de la investigación, desde su diseño hasta la construcción final del argumento. Las buenas prácticas que aquí se evidencian articulan preocupaciones antiguas de la reflexión metodológica cualitativa — especialmente, la consciencia sobre las operaciones propias de la investigación a medida que estas se llevan a cabo - con otras más recientes, como el nuevo acento en la transparencia. A continuación, identificamos recomendaciones inspiradas por nuestro estudio. Estas cubren áreas no abordadas en los catálogos de buenas prácticas ya existentes (Bennett y Checkel 2015; Trampusch y Palier 2016).

\section{Elegir la entrada apropiada}

Empezamos destacando los riesgos que surgen al elegir incorrectamente la entrada apropiada al process tracing para un caso dado de estudio. El primer tipo 
de riesgo implica perder una excesiva cantidad de tiempo, sin dudas una de las principales variables de ajuste en la práctica de la profesión. Por ejemplo, no vale la pena, en un afán inductivista, salir a campo demasiado pronto si contamos con trabajos de otros colegas que nos permiten pasar algún tiempo más en la oficina trabajando provechosamente de manera deductiva. Un investigador que elige la entrada inductiva, cuando las condiciones estaban dadas para una entrada deductiva, perderá valioso tiempo recolectando datos que luego verá que contribuían poco o nada para avanzar hacia la inferencia causal. Si hubiera usado desde el inicio las brújulas analíticas que ya existían, el investigador hubiera optimizado sus recursos. Por lo tanto, para minimizar los impactos de este tipo de riesgo, nuestro artículo sugiere que, si hay literatura establecida que desde el inicio puede identificarse con potencial analítico para explicar el caso de interés siempre es recomendable optar por la entrada deductiva. Ese camino minimiza los sesgos personales del investigador en el momento de "ingreso" al proyecto de investigación. En cambio, si en el momento de inicio el investigador no logra identificar con claridad un cuerpo de teoría que ilumine analíticamente el fenómeno empírico que lo intriga, o si ese cuerpo teórico sí es identificado pero el conocimiento del caso es todavía demasiado precario -debido a la ausencia de literatura secundaria de calidad y/o de datos confiables-, siempre es mejor optar por la entrada inductiva.

También existe un segundo tipo de riesgo, asociado a los atributos intrínsecos a cada entrada, aun cuando la decisión sobre la puerta de ingreso hubiera sido acertada. Por un lado, el principal riesgo que acecha a todo investigador que opta por la entrada inductiva surge de la tentación de incurrir en prácticas semejantes a las del data mining (minería de datos), es decir, extraer inductivamente un patrón de los datos desde el cual se inventa una teoría causal. Esta práctica "ensucia" los momentos deductivos posteriores: es muy probable que el "data miner cualitativo", contento con haber identificado un patrón interesante, tenga anteojeras a la hora de identificar teorías alternativas que quizá explicarían mejor los patrones observados. Asimismo, es probable que los posteriores momentos inductivos de la investigación también resulten sesgados: por ejemplo, el investigador puede caer en la reescritura de sus protocolos de entrevistas de un modo tendiente a inducir respuestas que, a su vez, hagan más convincente su teoría generada vía minería de datos. Por otro lado, el principal riesgo que acecha al investigador que arranca por la entrada deductiva es obnubilarse con una teoría que parece "funcionar" bien y, por lo tanto, no trabajar suficiente con las hipótesis alternativas. A falta de tiempo para hacer la iteración completa deducción-inducción-deducción-, es probable que se caiga en la tentación de no revisar críticamente la narrativa, forzando la teoría elegida a fin de complacer a jurados de tesis o réferis. ${ }^{9}$ 
La minimización del impacto que estos riesgos tienen sobre la calidad de la inferencia causal depende, a nuestro juicio, tanto de las prácticas individuales como de las intersubjetivas. Al nivel individual, requiere que los investigadores incrementen su nivel de conciencia respecto de en qué momentos de su trabajo están operando de manera deductiva y en cuales otros lo están haciendo de manera inductiva, lo que permite identificar a tiempo los riesgos más probables en cada una de las fases de la investigación y enfrentarlos analíticamente, sin negaciones. Al nivel intersubjetivo, en nuestra condición de réferis, directores y jurados de tesis, requiere ejercer cierta presión para que nuestros colegas expliciten los procedimientos inductivos y deductivos en sus caminos a la inferencia causal, sin por ello imponer corsés excesivos que conllevan un riesgo igualmente grave: en lugar de dominar el método, quedar dominados por él y, con ello, distraernos o incluso paralizarnos en el trabajo empírico que la ciencia política tiene por delante. ${ }^{10}$

\section{Maximizar la efectividad del trabajo de campo}

Los dos casos de este artículo sugieren una lección que puede ser especialmente valiosa para los investigadores jóvenes. En el diseño de los típicos cronogramas que se solicitan en los proyectos de investigación, para tesis de distintos niveles o proyectos, no suele contemplarse el hecho de que el process tracing más exitoso suele darse cuando el trabajo de campo no se hace "de un golpe" o en forma continua, sino que se realiza en varios rounds. Es recomendable entrecortar en el tiempo el trabajo empírico intensivo: solo ese modo discontinuo habilita que, efectivamente, la iteración entre momentos deductivos e inductivos que proponemos tenga el tiempo indispensable para desplegarse. Para dar un ejemplo, si el trabajo de una tesis doctoral requiere hacer process tracing en un país extranjero, es más probable que tres viajes de un mes produzcan mejores resultados que una única estadía larga de tres meses. Ese carácter discontinuo permite que los cuestionarios de las entrevistas y las estrategias de recolección de datos secundarios diseñados para los viajes segundo y tercero se adapten e incorporen las claves analíticas que surgen de los momentos deductivos entre viaje y viaje.

\section{Reconocer el punto de saturación para poner fin a la iteración}

¿Cuándo es adecuado dar por terminada la iteración de momentos inductivos y deductivos? Las iteraciones tienen rendimientos decrecientes y, al final del día, como ya señalamos, dependemos de la validación intersubjetiva para evaluar si no nos detuvimos demasiado pronto: que nuestros colegas, en su condición de evaluadores, dictaminen que efectivamente arribamos a un punto de saturación. 
Anticipando ese dictamen, los investigadores deberían continuar el trabajo hasta tanto se saturen (a) las hipótesis alternativas de las cuales deductivamente consideramos que es razonable extraer implicancias observables para explicar el resultado bajo estudio y (b) la capacidad de generar inductivamente explicaciones que es razonable considerar. Aquí, la lógica bayesiana ofrece una intuición de alto valor pragmático: uno se detiene cuando la repetición ocurre; esto es, un investigador debe dejar de perseguir toda fuente de evidencia cuando esto se vuelve tan repetitivo que, recolectar más evidencia de este tipo, tiene baja probabilidad de hacernos revisar nuestra estimación sobre cuán probable es que las hipótesis alternativas tengan alto poder explicativo (Bennett y Checkel 2015: 28). En línea con lo que destacamos a lo largo de este artículo, la honestidad intelectual es, en este sentido, determinante. Como sostienen Bennett y Checkel (2015: 30-1), "el process tracing concluyente es bueno, pero no todo buen process tracing es concluyente". Por lo tanto, si al final del trabajo de investigación persiste la incertidumbre respecto de si nuevas iteraciones entre inducción y deducción podrían alterar nuestras conclusiones, siempre es recomendable hacerlo explícito.

\section{Garantizar la honestidad intelectual y el control intersubjetivo}

Hoy las palabras de moda son transparencia, replicación y acceso a datos, a tal punto que muchas reflexiones sobre metodología cualitativa parecen haber estado originadas en complejos de inferioridad respecto a los métodos cuantitativos. Sin embargo, en vez de innovar en estándares que, supuestamente, pondrían nuestras inferencias causales a la altura de las que se realizan mediante métodos cuantitativos, resulta más productivo volver a cierta sabiduría originaria: por encima de todo, recuperar la importancia de la honestidad intelectual y, como señala Hall (2016: 28), la integridad de la investigación en la búsqueda sistemática de conocimiento sobre la política.

La recomendación, entonces, en términos de transparencia en process tracing, es, en primer lugar, mantener la tradición cualitativa de informar clara y extensamente sobre el proceso de recolección de datos. A este estándar clásico se suma la necesidad de mayor rigor y dedicación para acercarnos a la transparencia analítica: ser explícitos acerca de nuestra lógica de inferencia e identificar con precisión cómo contribuyen las piezas de evidencia a fortalecer nuestro argumento y a debilitar la plausibilidad de las hipótesis alternativas, proveyendo la información de contexto que orienta nuestra interpretación de la evidencia (ver Fairfield 2015: 47). En definitiva, nuestro trabajo lógico y empírico de process tracing debe dejar llano el camino para que otros colegas (con o sin interés intrínseco en nuestro caso), a partir de información que nosotros proporcionamos, puedan realizar lo que Büthe y Jacobs (2015: 57) denominan "replicación mental", que, sin replicar el estudio en sentido estricto, evalúe la solidez o debilidad de la conexión que nosotros establecemos entre observaciones procesuales y conclusiones. 
Avanzar hacia la transparencia, entendida de este modo, no requiere necesariamente procedimientos nuevos como los que pregona DA-RT (Data Access and Research Transparency) —cuya adopción, por otra parte, amenaza el desarrollo futuro de la investigación cualitativa (Hall 2016; Yashar 2016) — sino corregir sesgos existentes en los mecanismos de control intersubjetivo que ya tenemos. Dado que, en buena medida, la capacidad de la disciplina de aumentar los niveles de transparencia en la práctica del process tracing se juega durante la evaluación de manuscritos que hacen los editores y los réferis, en el contexto de América Latina hay amplio margen para mejorar. Específicamente, sería clave que las instituciones que evalúan las carreras académicas en la región tengan sintonía fina para diferenciar entre revistas que utilizan arbitrajes "blandos" y aquellas que imponen rigurosas evaluaciones de pares duros. En la búsqueda de mayores niveles de transparencia puede ser más importante garantizar el cumplimiento de las reglas ya existentes que la escritura de otras nuevas. La calidad analítica en el process tracing depende, en última instancia, de que los investigadores tengan incentivos para evitar los atajos de las revistas de arbitraje blando y opten por enviar sus trabajos a revistas cuyos réferis entiendan y exijan el cumplimiento de los criterios que identificamos en este artículo.

\section{CONCLUSIONES}

En este artículo nos hemos concentrado en la práctica del process tracing, herramienta adecuada para realizar inferencias causales a partir del análisis de un solo caso y, en particular, para poner en evidencia los mecanismos causales que conectan las variables independientes con los resultados que nos interesa explicar. Como este artículo es uno de los primeros en lengua castellana sobre process tracing, optamos por un foco pedagógico que permitiera discernir claramente lo nuevo que aporta este método, respecto de la tradicional práctica del estudio de caso y del método comparado. Al introducir el debate, establecimos un diálogo crítico con la tendencia dominante en la literatura en la frontera internacional de establecer una división dicotómica entre tipos deductivos e inductivos de process tracing.

La revisión de la literatura especializada y su confrontación con nuestra propia experiencia en análisis empírico con metodología cualitativa nos llevaron a elaborar una crítica a la literatura, que tiende a segmentar entre tipos de process tracing. Conociendo la tendencia a la fragmentación que impera entre quienes aplicamos métodos cualitativos, nos preocupan las querellas o desencuentros que puedan emerger entre partidarios de la inducción o la deducción. Mostramos, entonces, que no hay fundamento metodológico que sustente una división de este tipo, ya que la práctica de toda reconstitución de proceso conduce, después de la iteración de etapas inductivas y deductivas que fortalecen las hipótesis, a un mismo resultado: una narrativa causal plausible y persuasiva. La diferencia reside en el punto de partida, que depende del grado de desarrollo de la literatura sobre la pregunta de investigación al momento 
de iniciar cada trabajo específico. Por ejemplo aquí, el reconocimiento de una dinámica novedosa para la ciencia política - la paradójica situación de un gobierno que, debido al exponencial crecimiento de una política pública de subsidios por la cual recibe pocos aplausos de los votantes, se halla cada vez menos capaz de alinear el gasto público con sus prioridades políticas- nos motivó a adoptar una entrada inductiva. En cambio, la existencia de teorías ya avanzadas sobre los procesos de privatización de los sistemas de agua y sobre la misma "Guerra del agua", orientó hacia una entrada deductiva. Luego se sucedieron operaciones de investigación muy semejantes, en una secuencia que pronto terminó por converger.

Estos hallazgos de suma importancia tienen múltiples implicancias. En esta conclusión exploramos dos de ellas. En primer lugar, es de esperar que la creciente popularidad de este método lleve a la producción de una mayor cantidad de estudios de caso, dedicados al descubrimiento de diversos mecanismos causales, sean combinaciones o refinamientos de mecanismos ya conocidos o innovaciones completas. Esto plantea el riesgo de una agenda donde la acumulación de conocimiento podría verse dificultada. Si bien el uso disciplinado de conceptos compartidos previene que volvamos hacia los estudios idiosincráticos, es importante destacar que los estudios de caso deben siempre estructurarse con un espíritu comparativo y relacionarse con la literatura existente, ya sea desde un principio, cuando la entrada es deductiva, o en el transcurso de la investigación, cuando el punto de partida es inductivo. De hecho, hemos insistido en los dos estudios de caso en mostrar los momentos inductivos no como ateóricos sino, al contrario, como etapas en las que uno puede extraer de su conocimiento de la literatura algunas pistas para avanzar en la explicación del fenómeno de interés. Por su parte, el nuevo sano escepticismo sobre los métodos de Mill, como herramientas para la inferencia causal, y el consenso emergente sobre las ventajas del análisis intra-caso no deben ser interpretados como un llamado a abandonar la perspectiva comparada; por el contrario, en la medida en que la inferencia causal exitosa depende de la capacidad de minimizar los sesgos de variable omitida, los buenos estudios de caso serán aquellos que usen activamente la comparación para robustecer el análisis intra-caso, poniendo de manifiesto factores explicativos que las anteojeras de los estudios de tendencia idiosincrática tienden a ocultar.

El segundo punto tiene que ver con cómo se dan a conocer los resultados de investigación, independientemente de cómo fue su proceso de producción. Este artículo, al darle plena legitimidad a la entrada inductiva hacia la inferencia causal, invita a corregir ciertos sesgos dominantes en las prácticas de la ciencia política. Una ley no escrita, que parece regir los comportamientos de la mayoría de los investigadores de nuestra disciplina, es que las investigaciones - tesis doctorales, libros y artículos sometidos a arbitraje- de corte deductivo gozan de mayor probabilidad de ser aprobadas y/o publicadas que aquellas que explicitan su carácter inductivo. Esta tendencia parece constreñir, de manera más intensa, a las nuevas generaciones de investigadores en ciencia política. 
Por cierto, como nuestro artículo deja claro, no hay nada intrínsecamente problemático en la entrada deductiva hacia la inferencia causal. El problema que identificamos aquí surge cuando este modo de abordar la investigación comienza a ejercer un efecto corsé sobre los investigadores que tienen buenas razones para desarrollar estudios desde la entrada inductiva.

Esos constreñimientos no escritos suelen generar incentivos tendientes a vestir de ropajes deductivos a trabajos de investigación que son, en un primer momento, inductivistas, y que solo luego dan un paso hacia la evaluación deductiva de hipótesis. Esta práctica va en contra de las recomendaciones de transparencia que hoy son fundamentales para la investigación tanto cuantitativa como cualitativa. La retórica impostada del deductivismo hace daño al avance de investigaciones valiosas y termina por entorpecer el desarrollo ya iniciado en las corrientes cualitativas de la ciencia política a favor de la transparencia. Invitamos, entonces, a un pluralismo pragmático que responda no a la adscripción de un investigador a cierta práctica inductiva o deductiva, sino al estado del arte sobre las preguntas específicas que nos interesa estudiar.

\section{REFERENCIAS}

Bates, Robert. 1981. Markets and States in Tropical Africa: The Political Basis of Agricultural Policies. Berkeley: University of California Press.

Beach, Derek, y Rasmus Pedersen. 2013. Process Tracing Method. Foundations and Guidelines. Ann Harbor: The University of Michigan Press.

Bennett, Andrew. 2015. "Appendix: Disciplining Our Conjectures: Systematizing Process Tracing with Bayesian Analysis." En Process Tracing: From Metaphor to Analytic Tool, eds. Andrew Bennett y Jeffrey Checkel. Nueva York: Cambridge University Press, 276-298.

Bennett, Andrew, y Checkel, Jeffrey, eds. 2015. Process Tracing in the Social Sciences. From Metaphor to Analytic Tool. Nueva York: Cambridge University Press.

Brady, Henry, y David Collier. eds. 2010. Rethinking Social Inquiry: Diverse Tools, Shared Standards $2^{\text {nd }}$ ed. Lanham: Rowman and Littlefield.

Bril-Mascarenhas, Tomás, y Alison Post. 2014. "Policy traps y subsidios al consumo: la política de tarifas de servicios públicos en Argentina, 2002-2014." Desarrollo Económico 54 (213): 171-202.

Bril-Mascarenhas, Tomás, y Alison Post. 2015. "Policy Traps: Consumer Subsidies in Post-Crisis Argentina." Studies in Comparative International Development 50 (1): 98-120.

Büthe, Tim, y Alan Jacobs. 2015. "Conclusion: Research Transparency for a Diverse Discipline." Qualitative and Multi-Method Research 13 (1): 52-64.

Collier, David. 2011. "Understanding Process Tracing." PS: Political Science \& Politics 44 (4): 823-30.

Congreso Nacional de Bolivia. 1999. Ley N²029 de Servicios de Agua Potable y Alcantarillado Sanitario. Gaceta Oficial del Estado Plurinacional de Bolivia.

Dunning, Thad. 2012. Natural Experiments in the Social Sciences: A Design-Based Approach. Nueva York: Cambridge University Press.

Fairfield, Tasha. 2015. "Reflections on Analytic Transparency in Process Tracing Research." Qualitative and Multi-Method Research 13 (1): 47-51.

Fairfield, Tasha, y Andrew Charman. 2015. "Bayesian Probability: The Logic of (Political) Science Opportunities, Caveats, and Guidelines." Presentado en el Annual Meeting of the American Political Science Association, San Francisco. 
Gerring, John. 2007. Case Study Research. Principles and Practices. Nueva York: Cambridge University Press.

Hall, Peter. 2003. "Aligning Ontology and Methodology in Comparative Research." En Comparative Historical Analysis in the Social Sciences, eds. James Mahoney y Dietrich Rueschemeyer. Nueva York: Cambridge University Press, 373-404.

Hall, Peter. 2016. "Transparency, Research Integrity, and Multiple Methods." Newsletter of the Comparative Politics Organized Section of APSA 26 (1): 28-31.

Humphreys, Macartan, y Alan Jacobs. 2015. "Mixing Methods: A Bayesian Approach." American Political Science Review 109 (4): 653-73.

King, Gary, Robert Keohane, y Sidney Verba. 1994. Designing Social Inquiry: Scientific Inference in Qualitative Research. Princeton: Princeton University Press.

Lorentzen, Peter, Taylor Fravel, y Jack Paine. 2017. “Qualitative Investigation of Theoretical Models: The Value of Process Tracing." Journal of Theoretical Politics 29 (3): 467-491.

Mayaux, Pierre-Louis. 2012. "La privatisation et ses contestataires: réformes et conflits dans les politiques d'accès à l'eau potable à Carthagène, La Paz, Cochabamba et Campo Grande, 1980-2010." Tesis de Doctorado, Sciences Po.

McAdam, Douglas, Sidney Tarrow, y Charles Tilly. 2008. "Methods for Measuring Mechanisms of Contention." Qualitative Sociology 31 (3): 307-331.

O’Donnell, Guillermo. 1978. "Tensiones en el Estado burocrático-autoritario y la cuestión de la democracia." Documento del Centro de Estudios de Estado y Sociedad (CEDES) N ${ }^{\circ}$ 11. Ciudad Autónoma Buenos Aires: CEDES.

Przeworski, Adam, y Henry Teune. 1970. The Logic of Comparative Social Inquiry. Nueva York: John Wiley.

Ragin, Charles. 2007. La construcción de la investigación social. Introducción a los métodos y su diversidad. Bogotá: Siglo del Hombre Editores (SHE).

Sartori, Giovanni. 1970. "Concept Misformation in Comparative Politics." American Political Science Review 64 (4): 1033-53.

Tansey, Oisín. 2007. "Process Tracing and Elite Interviewing: A Case for Non-Probability Sampling." PS: Political Science \& Politics 40 (4): 765-72.

Tilly, Charles, y Sidney Tarrow. 2007. Contentious Politics. Boulder: Paradigm Publishers

Timmermans, Stefan, e Iddo Tavory. 2012. "Theory Construction in Qualitative Research: From Grounded Theory to Abductive Analysis." Sociological Theory 30 (3), 167-186.

Trampusch, Christine, y Bruno Palier. 2016. "Between X and Y: How Process Tracing Contributes to Opening the Black Box of Causality." New Political Economy 21 (5): 437-454.

Weaver, Kent. 1986. "The Politics of Blame Avoidance." Journal of Public Policy 6 (4): 371-98.

Weyland, Kurt. 1996. "Risk Taking in Latin American Economic Restructuring: Lessons from Prospect Theory." International Studies Quarterly 40 (2): 185-207.

Yashar, Deborah. 2016. "Editorial Trust, Gatekeeping, and Unintended Consequences." Newsletter of the Comparative Politics Organized Section of APSA 26 (1): 57-64.

Yom, Sean. 2015. "From Methodology to Practice: Inductive Iteration in Comparative Research." Comparative Political Studies 48 (5): 616-44.

Tomás Bril-Mascarenhas es Ph.D. en Political Science por University of California-Berkeley. Es profesor en la Escuela de Política y Gobierno de la Universidad Nacional de San Martín (UnSAM, Argentina). Ha publicado, junto a Alison Post, "Policy Traps: Consumer Subsidies in Post-Crisis Argentina" (Studies in Comparative International Development) y, con Aldo Madariaga, "Business Power and the Minimal State: The Defeat of Industrial Policy in Chile" (en prensa, Journal of Development Studies). Su investigación anterior sobre sistemas partidarios subnacionales comparados fue publicada en Desarrollo Económico. Se especializa en economía política comparada, con foco en la interacción entre actores del empresariado y los estatales, en procesos de política pública en Argentina, Brasil y Chile. Correo electrónico: tombril@berkeley.edu

Antoine Maillet es doctor en Ciencia Política por la Universidad Católica de Chile y Sciences Po. Es profesor asistente en el Instituto de Asuntos Públicos de la Universidad de Chile e investigador adjunto del Centro de Estudios de Conflicto y Cohesión Social (Coes). Su investigación sobre las 
trayectorias de las políticas neoliberales en Chile ha sido expuesta en los artículos "Más allá del 'modelo' chileno: una aproximación multi-sectorial a las relaciones Estado-mercado" (Revista de Sociología e Política) y "Variedades de neoliberalismo. Innovación conceptual para el análisis del rol del Estado en los mercados" (Revista de Estudios Políticos). Correo electrónico: antoinemaillet@iap. uchile.cl

Pierre-Louis Mayaux es doctor en Ciencia Política por Sciences Po. Es investigador del Centro Internacional de Investigación en Agronomía para el Desarrollo (CIRAD), en residencia en la Escuela de Gobernanza y Economía de Rabat (Marruecos). Su trabajo aborda las políticas públicas del agua y la legitimación de la acción pública. Sus principales publicaciones son "Amérique Latine: les réformes de marché en question" (Reoue Internationale de Politique Comparée, 2012, con Yves Surel) y "Encourager la subversion: recomposition de l'État et décollectivisation des terres publiques du BasChéliff, Algérie" (Politique Africaine, 2015, con Hichem Amichi y Sami Bouarfa). Correo electrónico: pierre-louis.mayaux@cirad.fr 